Sandermann, W.; Rothkamm. M.: Über die Bestimmung der pHWerte von Handelshölzern und deren Bedeutung für die Praxis. Holz. Roh-Werkstoff 17 (1959) 433-440

Sergejeva, V. N.; Miljutina. S. V. (1960), zitiert aus Fengel, D.: Wegener, G.: Wood chemistry, ultrastructure, reactions. Walter de Gruyter. Berlin, New York 1984

Schneider, A.: Orientierende Vergleichsuntersuchungen über das Sorptionsverhalten mitteleuropäischer Baumrinden und Hölzer. Holz Roh-Werkstoff 36 (1978) 235 -239

Schneider, A; Rusche. H.: Sorptionsverhalten von Buchen- und Fichtenholz nach Wärmeeinwirkung in Luft und im Vakuum. Holz Roh-Werkstoff 31 (1973) 313.319

Schwarz, H. G.: Cement-bonded boards in Malaysia. Proceeding of the International Conference on Fiber and Particleboards Bonded with Inorganic Binders. October 24- 26, 1988. University of Idaho. Moslemi, A. A. (ed.). Idaho 1989
Skatar, C.: Water in Wood. Syracause University Press. Syracause. 218 pp 1972

Standke, W.; Schneider, A.: Untersuchungen über das Sorptionsverhalten des Bast- und Borkeanteils verschiedener Baumrinden. Holz Roh-Werkstoff 39 (1981) 489-493

Stiasny, E.: in "Der Gerber" S. 187 zitiert aus Gnamm, H.: Die Gerbstoffe und Gerbmittel. Wissenschaftliche Verlaggesellschaft mbH. Stuttgant 1905

Wise, E. L. (1946) im Merck AG (Hrsg): Chemisch-technische Untersuchungsmethoden für die Zellstolf- und Papierfabrikation. Verlag Chemie GmbH. Weinheim 1957

Wissing, A.: The utilization of bark II: Investigation of the Stiasny reaction for the precipitation of polyphenols in pine bark cxtractives. Svensk. Papperstidning 58 (20) (1955) 745750

\section{Untersuchungen zur Ermittlung von Kenn- linien für die Bestimmung des Anteils an Phenolharz in Spangemischen}

\section{P. Niemz; O. Wienhaus}

Technische Universität Dresden. Mommsenstrabe 13 , O-8027 Dresden

\section{B. Stölken}

Landes[orschungsanstalt für Landwirtschaft und Fischerei,

Dorfplatz 1, O-2601 Gülzow

Subject: The NIR-absorption (diffuse reflection mode) at wavelength of 1428,1870 , and $2020 \mathrm{~nm}$ are used to characterize the ratio of phenole resins in chip mixtures. Linear combination showed high correlation between experimental and theoretical values.

Material und Methode: Späne aus Kiefernholz wurden mit 5\%,8\% $10 \%, 12 \%, 15 \%, 20$ und $25 \%$ Phenolharz (Plastacol $2062 \mathrm{H}$, Plasta Erkner GmbH) unter Laborbedingungen nach dem Sprüh- und Umwälzverfahren beleimt. Die Spanproben wurden mit einer Schlagmühle QC 114 der Fa. MIM. Budapest mit einem Sieb 13/100 nachzerkleinert. Für die Aufnahme der Spektren diente das NIRSpektrometer Infrapid 61 der Fa. MIM, Budapest. Nach der von Niemz et al. (1992) beschriebenen Technik wurden aus den NIRSpektren die Wellenlängen ermittelt, bei denen eine starke Absorption auftrat und danach eine lineare Regression zwischen dem Festharzanteil und der diffusen Reflexion (R) bzw. der optischen Dichte (D) berechnet.

Ergebnisse: 1. Als gut geeignet für die Regression erwiesen sich die Wellenlängen $1870 \mathrm{~nm}, 1428 \mathrm{~nm}, 2020 \mathrm{~nm}$. 2. Unter Einbeziehung der 3 o. g. Wellenlängen ergab sich folgende Regressionsgleichung für den Festharzanteil:

$\mathrm{FH}=48,76+1935,4 \times \mathrm{Ci}-873,8 \times \mathrm{C} 2-704,3 \times \mathrm{C} 3(\mathrm{R}=0,987)$
$\mathrm{Cl}=$ Reflexion bei Wellenlänge $1878 \mathrm{~nm}, \mathrm{C} 2=$ Reflexion bei Wellenlänge $1428 \mathrm{~nm}, \mathrm{C} 3=$ Reflexion bei Wellenlänge $2020 \mathrm{~nm}$

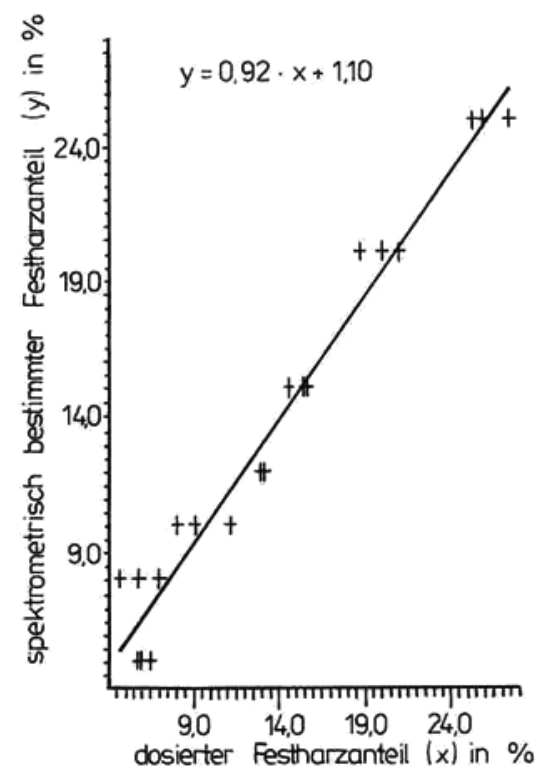

Bild 1. zeigt die Korrelation zwischen dosiertem Festharzanteil und dem optisch bestimmten

Niemz, P. et al: Orientierende Untersuchungen zur Anwendung der NIR - Spekroskopic Jür die Beurteilung des Mischungsverhältnisses Laub-/Nadelholz und des Klebstoffanteils in Spangemischen. Holz Roh-Werkstoff 50 (1992) 2528 